\title{
Philosophiques
}

\section{Rationalité, humanité, normativité}

\section{Sarah Stroud}

Volume 31, numéro 2, automne 2004

URI : https://id.erudit.org/iderudit/009816ar

DOI : https://doi.org/10.7202/009816ar

Aller au sommaire du numéro

Éditeur(s)

Société de philosophie du Québec

ISSN

0316-2923 (imprimé)

1492-1391 (numérique)

Découvrir la revue

Citer ce document

Stroud, S. (2004). Rationalité, humanité, normativité. Philosophiques, 31(2),

405-408. https://doi.org/10.7202/009816ar d'utilisation que vous pouvez consulter en ligne.

https://apropos.erudit.org/fr/usagers/politique-dutilisation/ 
il accepte ce qu'il nomme «l'argument sophistique " : s'il est raisonnable de parfois agir selon son meilleur jugement, c'est parce que parfois cette action se trouve en fait - aux yeux de tiers - rationnelle. Mais la probabilité que nous nous trouvions dans de telles situations est très faible.

À tout le moins, on ne peut l'encourager que dans beaucoup moins de $25 \%$ des cas, et elle ne pourrait donc être rationalisée que par une stratégie évolutionnairement stable qui soit faite d'une mixture de probabilités. Je ne suis pas un adepte de l'utilisation du formalisme des probabilités dans ces domaines, mais, à tout le moins, il serait raisonnable de ne pas ranger toutes les normes morales auxquelles on peut penser au même rang de normalité. Ce sont des réflexions sur ces pondérations - pondérations des arguments et pondération des normes - qui me semblent faire défaut dans la méthode de Ruwen Ogien, alors même que ses conclusions montrent que la plupart du temps il pratique sans la thématiser une telle pondération.

\section{Rationalité, humanité, normativité}

\section{SARAH STROUD McGill}

Ce livre plein de sagesse, de modération, de bon sens donne au lecteur le sentiment agréable de participer à un dialogue philosophique avec l'auteur. Dans l'esprit d'une telle conversation, j'aimerais poser ici quelques questions suscitées par certains des essais colligés dans Le rasoir de Kant. Elles ont pour but de poursuivre le dialogue avec Ruwen Ogien que ses réflexions dans le livre semblent solliciter.

\section{Le rationnel et le raisonnable; la partialité et l'impartialité}

Ogien lie le rationnel à la partialité et le raisonnable à l'impartialité. Ce premier article du recueil soulève donc la question de la relation entre la partialité et l'impartialité. J'aimerais souligner une remarque importante de l'auteur à ce sujet. Bon nombre de critiques de l'impartialité en éthique sont en fait dirigées contre l'idée d'un "principe substantiel» (32) d'impartialité qui nous ordonnerait de traiter chaque personne de la même façon ${ }^{1}$. Mais, comme le mentionne Ogien, ce n'est pas le seul rôle que peut jouer l'impartialité dans un système éthique; et il est frappant de constater que peu de théories morales courantes contiennent un tel principe de premier ordre. Passons donc à l'idée que l'impartialité est plutôt un point de vue à partir duquel il est possible de choisir, d'adopter, d'endosser ou de justifier des principes de premier ordre. Ogien remarque que le fait d'occuper une telle perspective n'implique pas

1. Voir par exemple John Cottingham, «Ethics and Impartiality ", Philosophical Studies 43 (1983), 83-99; John Kekes, "Morality and Impartiality ", American Philosophical Quarterly 18 (1981), 295-303; Lawrence Blum, Friendship, Altruism, and Morality (Boston : Routledge \& Kegan Paul, 1980), ch. III. 
que les principes choisis seront forcément impartiaux : «Il est parfaitement concevable que des principes partialistes soient recommandés à partir d'un point de vue impartial» (33). J'admets que ceci est concevable, mais j'aimerais savoir s'il y a des raisons concrètes de penser que c'est effectivement le cas. J'ai l'impression qu'Ogien croit qu'il y en a, car il parle à plusieurs reprises de "dériver» le rationnel (le partial) du raisonnable (l'impartial), et ce mot semble indiquer un rapport plus étroit entre les deux qu'une simple absence d'incompatibilité. (Dériver un théorème de certaines prémisses, ce n'est pas montrer que celui-là est compatible avec celles-ci, c'est montrer que celui-là est une conséquence logique de celles-ci.) Cette formulation suggère plutôt que la perspective impartiale exige l'adoption de principes partialistes, que ceuxci seraient des conséquences ou des corollaires de toute procédure impartiale de sélection. Certaines formes de l'utilitarisme réclament que cela soit le cas ${ }^{2}$, mais à part ces tentatives, je vois mal les raisons pour lesquelles on aurait le droit de parler de dériver le partial de l'impartial comme le fait Ogien.

\section{La rationalité de la faiblesse de la volonté}

Ogien présente trois arguments en faveur de la proposition que la faiblesse de la volonté n'est pas nécessairement irrationnelle. Il dit trouver le troisième argument dans Davidson, mais personnellement je ne trouve pas d'argument pour cette conclusion dans "How is Weakness of the Will Possible?". L'argument que décrit Ogien établirait, au plus, que la faiblesse de la volonté n'implique aucune contradiction logique (à moins que l'agent ait adopté le principe de continence). Mais ceci n'équivaut pas à dire que la faiblesse de la volonté n'est pas forcément irrationnelle. Ogien semble assimiler les deux quand il dit «qu'il n'y a aucune contradiction logique, aucune irrationalité intrinsèque» (64; voir aussi 70) dans le fait d'agir pour une raison tout en croyant que les raisons pour agir autrement sont meilleures. Mais ce n'est que si on suppose que la contradiction logique constitue la seule forme d'irrationalité que ces deux formulations sont équivalentes. Selon moi, Davidson (même le Davidson de "How is Weakness of the Will Possible?") n'endosserait pas cette proposition. Je ne la trouve pas très plausible, d'ailleurs ${ }^{3}$. Je passe donc aux deux premiers arguments présentés par Ogien dans cet article.

Comme il le remarque, ces deux arguments se ressemblent $:$ ils ont la même structure logique (67), et ils sont exposés aux mêmes objections (69).

2. Voir par exemple Brad Hooker, Ideal Code, Real World (Oxford : Clarendon Press, 2000), 108-111, 136-141; et Frank Jackson, «Decision-theoretic Consequentialism and the Nearest and Dearest Objection ", Ethics 101 (1991), 461-482. Ces textes maintiennent que la partialité est nécessaire à l'obtention des résultats qui sont meilleurs du point de vue impartial.

3. Par exemple, elle semble exclure d'emblée ce que Christine Korsgaard appelle «true irrationality ", c'est-à-dire le fait de ne pas être motivé à prendre les moyens nécessaires à ses fins. Ceci est un type d'irrationalité spécifiquement pratique, qui n'implique aucune contradiction logique; ce ne serait donc pas une véritable forme d'irrationalité selon la proposition en question. Voir Christine M. Korsgaard, "Skepticism about Practical Reason ", dans son Creating the Kingdom of Ends (Cambridge : Cambridge University Press, 1996), 318-319. 
Il me semble que de tels arguments font l'erreur de ne pas distinguer entre faire un choix rationnel, ou choisir rationnellement, d'un côté, et faire ce qu'on a en fait le plus de raisons de faire ${ }^{4}$, de l'autre. Ces deux arguments commencent en effet par l'observation qu'il y a des cas où le meilleur jugement d'un agent est erroné et ne correspond pas à ce qu'il a véritablement le plus de raisons de faire. Dans ces cas, il est évident que l'agent ne fera pas ce qu'il a en fait le plus de raisons de faire que s'il agit à l'encontre de son meilleur jugement. Il est donc tout à fait possible qu'en manifestant la faiblesse de la volonté l'agent réussira quand même à choisir la "bonne » action.

Je suis d'accord avec cette démonstration, mais je n'y trouve pas d'argument faisant valoir que son choix n'est pas un exemple d'irrationalité. Si on sépare les deux notions que je viens de mentionner plus haut - tout comme on le ferait pour des notions analogues, telles que vérité et justification (65) - le fait d'arriver à la réponse correcte (pour ainsi dire) n'exempte pas l'agent de l'accusation d'irrationalité en ce qui concerne le processus de raisonnement qu'il a suivi pour y parvenir. (Comparez : quelqu'un arrive à une croyance vraie par une erreur logique dans son raisonnement. Est-ce que cela veut dire que c'est une croyance rationnelle?) Faire cette distinction n'équivaut pas à dire que les critères de la rationalité sont purement internes (comparer 65-67, 69). Il s'agit plutôt de maintenir que faire, comme l'akratique, un choix allant à l'encontre de son propre raisonnement constitue une condition suffisante pour l'irrationalité Une si grosse erreur de procédure dans le raisonnement pratique justifie une accusation d'irrationalité, quels que soient les mérites du choix qui en résulte.

\section{«Devoir implique pouvoir » et la portée du normatif}

Je trouve fort intéressante la proposition selon laquelle «devoir implique pouvoir » n'exprimerait pas une vérité logique ou conceptuelle, mais reposerait plutôt sur un principe moral substantiel, qu'Ogien appelle le principe d'humanité. Avant de passer à autre chose, deux remarques rapides sur cet essai. Je n'ai pas bien suivi les raisons pour lesquelles Ogien rejette une interprétation pragmatique du lien entre «devoir» et "pouvoir», et je ne comprends pas tout à fait son pessimisme à l'égard des théories morales (77). Il me semble qu'entre le principe d'humanité (qui exclut les normes trop cruelles) et le principe de parcimonie (qui élimine les normes inutiles), il nous reste une grande marge de manœuvre.

Mais j'aimerais soulever un autre problème. Si « devoir implique pouvoir » n'est pas une vérité logique ou nécessaire, notez qu'il n'y a en principe aucune objection logique (seulement une objection morale) à l'application de notions normatives (comme «devoir») à ce qui est impossible. Comme le

4. T. M. Scanlon souligne l'importance de distinguer entre «claims about rationality» et "substantive claims about what reasons there are"; voir What We Owe to Each Other (Cambridge (Mass.) : Harvard University Press, 1998), 23 ss. Selon Scanlon, le premier concerne « the form that our thinking must take», le second spécifie "what we have most reason to do" (30).

5. Comparer Philippa Foot, Natural Goodness (Oxford : Clarendon Press, 2001), 74-76. 
dit Ogien, "des normes qui nous demandent l'impossible ne sont pas inconcevables" (90). Parallèlement, l'application des normes à ce qui n'est pas intentionnel, ou à ce qui ne peut pas être objet de choix ou de volonté, ne poserait aucun problème logique ou conceptuel. Mais ces conséquences semblent nuire à l'argument du cinquième article, «Le normatif et l'évaluatif ». Ogien y donne onze raisons de "séparer nettement» les évaluatifs des normatifs (95), mais il privilégie l'une d'elles (97), à savoir les différents domaines d'application des évaluatifs et des normatifs. Il propose effectivement que la portée des normatifs soit plus étroite que celle des évaluatifs : contrairement aux évaluatifs, «les normatifs s'appliquent aux actions humaines intentionnelles exclusivement» (96), ou, plus précisément, «à ce qui peut être objet de choix ou de volonté, à ce qui n'est ni nécessaire ni impossible» (119). Ogien trouve cette différence entre les deux catégories «1[a] plus intéressant[e] ou 1[a] plus riche de conséquences» (119) de toute la liste qu'il propose, et elle joue un rôle important dans son rejet de la théorie du «tout normatif » à la fin de l'article. L'argument d'Ogien repose donc en grande partie sur ce critère de distinction entre les normatifs et les évaluatifs.

Si on suit la proposition citée plus haut sur les limites du domaine du normatif, ce serait une erreur de catégorie que d'appliquer les normatifs à des choses qui ne sont pas des actions humaines intentionnelles, ou qui sont impossibles, ou qui ne sont pas objet de choix ou de volonté. Mais l'argument de l'essai précédent semble amoindrir cette proposition. Comme on l'a déjà remarqué, Ogien ne voit aucun obstacle logique ou conceptuel à l'application des normes en dehors de ces conditions. Elles s'appliquent en principe à ce qui n'est pas objet de choix ou de volonté : une telle application ne manifesterait aucune erreur de catégorie, même s'il y a des raisons morales de ne pas endosser de telles normes. Ce point, cependant, semble effacer la distinction qu'Ogien souhaite entre le domaine d'application des normatifs et celui des évaluatifs.

\section{Réponses à mes critiques}

\section{RUWEN OGIEN \\ CNRS \\ ruwen@wanadoo.fr}

\section{Réponse à Charles Larmore}

Dans un commentaire lumineux, Charles Larmore me reproche de m'en prendre dans mon introduction à une sorte de "normativisme généralisé " qui n'est défendu officiellement par personne, mais il précise très gentiment que ce n'est pas ce que je propose en fait dans les essais qui suivent, ce qui fait que cette objection ne lui paraît finalement pas trop cruciale. Je me permettrai cependant de lui faire remarquer qu'il y a pas mal de relativistes, de conventionnalistes, de constructivistes, d'anti-réalistes radicaux ou même de "réalistes internes» qui aspirent à éliminer la dichotomie des faits et des valeurs et insistent sur le caractère socialement ou moralement «construit» des faits. Ils ne 